\title{
Early Cerebral Circulatory Disturbance in Patients Suffering Subarachnoid Hemorrhage Prior to the Delayed Cerebral Vasospasm Stage: Xenon Computed Tomography and Perfusion Computed Tomography Study
}

\author{
Mitsuru Honda, ${ }^{1}$ Shigeru SASE, ${ }^{4}$ Kyosuke YOKOTA, ${ }^{1}$ Ryo ICHIBAYASHI, ${ }^{1}$ \\ Katsunori YOSHIHARA, ${ }^{1}$ Yoshihito SAKATA, ${ }^{1}$ Hiroyuki MASUDA, ${ }^{2}$ \\ Hiroyuki UEKUSA, ${ }^{2}$ Yoshikatsu SEIKI, ${ }^{2}$ and Taichi KISHI ${ }^{3}$ \\ Departments of ${ }^{1}$ Critical Care Center and ${ }^{2}$ Neurosurgery, \\ Toho University Medical Center Omori Hospital, Tokyo; \\ ${ }^{3}$ Department of Education Planning and Development, School of Medicine, \\ Faculty of Medicine, Toho University, Tokyo; \\ ${ }^{4}$ Anzai Medical Co., Ltd., Tokyo
}

\begin{abstract}
Subarachnoid hemorrhage (SAH) causes dynamic changes in cerebral blood flow (CBF), and results in delayed ischemia due to vasospasm, and early perfusion deficits before delayed cerebral vasospasm (CVS). The present study examined the severity of cerebral circulatory disturbance during the early phase before delayed CVS and whether it can be used to predict patient outcome. A total of 94 patients with SAH underwent simultaneous xenon computed tomography (CT) and perfusion CT to evaluate cerebral circulation on Days 1-3. Cerebral blood flow (CBF) was measured using xenon CT and the mean transit time (MTT) using perfusion CT and calculated cerebral blood volume (CBV). Outcome was evaluated with the Glasgow Outcome Scale (good recovery [GR], moderate disability [MD], severe disability [SD], vegetative state [VS], or death [D]). Hunt and Hess (HH) grade II patients displayed significantly higher CBF and lower MTT than HH grade IV and V patients. HH grade III patients displayed significantly higher CBF and lower MTT than HH grade IV and V patients. Patients with favorable outcome (GR or MD) had significantly higher CBF and lower MTT than those with unfavorable outcome (SD, VS, or D). Discriminant analysis of these parameters could predict patient outcome with a probability of $\mathbf{7 4 . 5 \%}$. Higher HH grade on admission was associated with decreased CBF and CBV and prolonged MTT. CBF reduction and MTT prolongation before the onset of delayed CVS might influence the clinical outcome of SAH. These parameters are helpful for evaluating the severity of SAH and predicting the outcomes of SAH patients.
\end{abstract}

Key words: subarachnoid hemorrhage, acute cerebral circulation disturbance, xenon computed tomography, perfusion computed tomography

\section{Introduction}

Subarachnoid hemorrhage (SAH) remains of considerable concern to neuroclinicians due to its refractory high morbidity and mortality. Delayed cerebral vasospasm (CVS) is one of the major risk factors contributing to death and disability in $\mathrm{SAH}$ patients. Considerable efforts have been made to understand the mechanisms responsible and to find

Received July 21, 2011; Accepted November 19, 2011 ways to prevent and treat delayed CVS. None of these issues have been resolved. However, early brain injury makes significant contributions to patient outcomes and might have an even more significant effect on outcome than delayed CVS. In fact, the outcome of SAH is known to be most significantly correlated with the initial clinical presentation of the patient and decreased cerebral blood flow (CBF). ${ }^{23)}$ Several factors can cause decreased CBF: intracranial hypertension, hydrocephalus, intracerebral hematoma, vasospasm, brain edema, and the direct toxic effects of SAH. Previous clinical in- 
vestigations have detected reduced CBF during the acute stage of SAH.6,11,20) Recently, computed tomography (CT) perfusion imaging has been used to assess patients with delayed CVS. ${ }^{12,25,28)} \mathrm{A}$ number of hemodynamic parameters are available for CT perfusion imaging. One such parameter, the mean transit time (MTT), is defined as the mean time required for blood to perfuse through a region of tissue, and is highly sensitive to hemodynamic disturbances. There have been several investigations of cerebral circulatory disturbance during the delayed CVS phase using MTT.4,14,25,28) MTT was identified as the most sensitive perfusion parameter for detecting delayed CVS. ${ }^{28)}$ However, few investigations have attempted to explain circulatory disturbances using MTT and cerebral blood volume (CBV) during the early phase before delayed CVS.

The present study investigated the relationship between hemodynamic parameters, neurological grade on admission, and patient outcome in the early phase (Days 1-3) before delayed CVS. MTT, CBF, and CBV were measured using perfusion $\mathrm{CT}$ and xenon-enhanced CT (Xe-CT) to evaluate utility as predictors of the severity of early circulatory disturbance and outcome after SAH.

\section{Methods and Subjects}

Ninety-four patients, 39 men and 55 women aged from 31 to 86 years (mean: 61.2 years), admitted to our hospital with SAH were prospectively enrolled. The clinical characteristics are shown in Table 1. All 94 patients underwent surgical clipping or endovascular coiling for ruptured aneurysms. SAH was verified by CT on admission in all patients. Patients who were admitted within 24 hours of suffering SAH were included in this study. The exclusion criteria for the present study were patients with SAH due to a cause other than a ruptured aneurysm, patients younger than 18 years of age, patients in a state of cardiopulmonary arrest on arrival, patients with SAH due to a ruptured posterior circulation aneurysm, patients who did not undergo treatment, and patients with surgical iatrogenic damage. The clinical statuses of all patients were recorded on admission according to the Hunt and Hess $(\mathrm{HH})$ grading system. ${ }^{10)}$ Written informed consent for the study was obtained from all patients or guardians, and the study protocol was approved by the ethics committee of our institute. All patients were treated according to a standardized protocol consisting of absolute bed rest and intravenous administration of sedative agents until the aneurysm was treated. The postoperative management protocol included mild hypervolemic therapy via the in-
Table 1 Patient characteristics

\begin{tabular}{lc}
\hline Characteristic (total =94) & Number of patients (\%) \\
\hline Women & $55(59 \%)$ \\
Mean age in years (range) & $61.2(31-86)$ \\
Hunt and Hess grade & \\
II & $26(28 \%)$ \\
III & $35(37 \%)$ \\
IV & $25(27 \%)$ \\
V & $8(9 \%)$ \\
Fisher group & \\
2 & $6(6 \%)$ \\
3 & $15(16 \%)$ \\
4 & \\
Aneurysm location & $28(30 \%)$ \\
ICA & $29(31 \%)$ \\
MCA & $37(39 \%)$ \\
ACA & $75(80 \%)$ \\
Surgical procedure & $19(20 \%)$ \\
clipping & \\
endovascular &
\end{tabular}

ACA: anterior cerebral artery territory, ICA: internal carotid artery territory, MCA: middle cerebral artery territory.

travenous administration of low molecular weight dextran when necessary. Dobutamine and nicardipine were given to most patients during the risk period for delayed CVS. Continuous cisternal, ventricular, or lumbar external drainage was employed to control intracranial pressure (ICP). Xe-CT and perfusion $\mathrm{CT}$ were simultaneously performed immediately after postoperative CT on Days 1-3. All studies were performed after the ruptured aneurysm had been treated.

The neurological conditions of all patients were recorded by the attending neurosurgeons throughout their hospital stay. The following information was recorded for each patient: sex, age, clinical condition, and aneurysm location. Outcome was assessed at 3 months after onset according to the Glasgow Outcome Scale and recorded as good recovery (GR), moderate disability (MD), severe disability (SD), vegetative state (VS), or death (D).

After standard CT, Xe-CT and perfusion CT were carried out continuously using an X-Vigor scanner (Toshiba, Tokyo). As measuring CBF using perfusion CT is not a quantitative method, we measured CBF using Xe-CT. We measured MTT by perfusion CT and calculated CBV using the AZ-7000W98 computer system (Anzai Medical, Tokyo). CBV maps were produced by multiplying the $\mathrm{CBF}$ and MTT according to the central volume principle: $\mathrm{CBV}=\mathrm{CBF}$ $\times$ MTT. ${ }^{15)} \mathrm{CBF}, \mathrm{MTT}$, and CBV maps were created at the level of the basal ganglia (Fig. 1). 

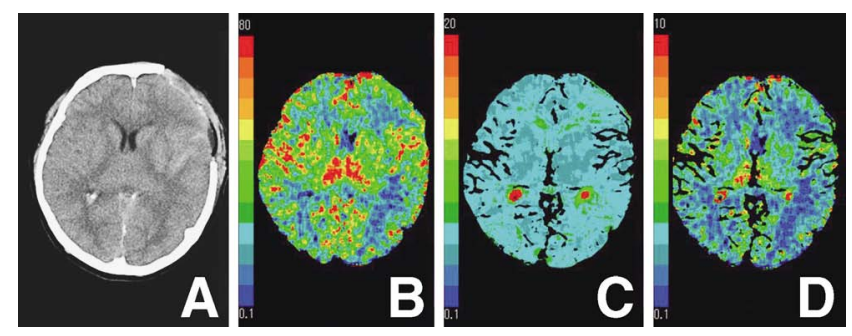

Fig. 1 Computed tomography scan (A), cerebral blood flow map (B), mean transit time map (C), and cerebral blood volume map (D).

In Xe-CT, the patients inhaled $30 \%$ stable xenon for 4 minutes (wash-in), followed by 5 minutes of desaturation (wash-out), using a xenon gas inhalation system (AZ-725; Anzai Medical). CT scans were performed at 1-minute intervals, and 3 levels of the brain (centered on the basal ganglia) were imaged in each scan by moving the CT table. A $512 \times 512$ matrix and a $10-\mathrm{mm}$ slice thickness were used. The exposure factors were $120 \mathrm{kV}(\mathrm{p})$ and $200 \mathrm{~mA}$, and the scan time was 2 seconds. The pixel size was $0.469 \mathrm{~mm} \times 0.469 \mathrm{~mm}$. CBF maps were created using the resultant CT images and end-tidal xenon data. Unweighted filtering over a $9 \times 9$ area was performed on the original CT images prior to computation. A $\lambda$-guided calculation method ${ }^{21)}$ was applied using end-tidal xenon as a substitute for arterial xenon. On a pixel-by-pixel basis, CBF (ml/100 $\mathrm{g} / \mathrm{min}$ ) and $\lambda$, the xenon brain-blood partition coefficient, were calculated according to the GaussNewton method using the Kety autoradiographic equation.

Perfusion CT was performed just after the standard CT and Xe-CT. Twenty dynamic conventional scans were obtained in a plane $50 \mathrm{~mm}$ above the orbitomeatal orientation, which included the basal ganglia; the thalamus; and parts of the anterior, middle, and posterior cerebral arterial territories. Thirty milliliters of non-ionic contrast material (Iomeron 300; Eisai Co., Ltd., Tokyo) were injected into the central vein through a 16-gauge cannula with a power injector (Auto Enhance A-60; Nemoto Kyourindo, Tokyo) at an infusion rate of $9 \mathrm{ml} / \mathrm{sec}$. CT scanning began 5 seconds after the start of the injection. CT scans were performed at intervals of 2 seconds for 30 seconds, and subsequently at 6 -second intervals for 30 seconds. The resultant CT images were used to calculate MTT. On a pixel-by-pixel basis, the time course of the change in CT enhancement (time-density curve) was fitted to the gamma variate using the Gauss-Newton method, and the distance between two inflection points (maximum upward slope and maximum downward slope) was calculated as the MTT value. ${ }^{18)}$ Then, CBV maps were created by multiplying CBF and MTT according to the central volume principle: $\mathrm{CBV}=\mathrm{CBF} \times \mathrm{MTT}{ }^{15)}$

All parameters are presented as the mean \pm standard deviation. The value of each parameter was averaged using regions of interest in both hemispheres at the basal ganglia level (Fig. 1). The ttest, analysis of variance, and multiple comparisons analysis were used to assess the differences between groups. Discriminant analysis was used to examine the power of the parameters to predict outcomes. Statistical significance was set at $p<0.05$ for differences between patient groups. All statistical calculations were performed with a personal computer using a statistical software package (SPSS, version 12.0; SPSS Japan, Tokyo).

\section{Results}

The relationships between neurological grade and the hemodynamic parameters CBF, MTT, and CBV were examined. CBF decreased significantly as the neurological grade on admission increased, indicating significant grade dependence $(\mathrm{p}<0.05$, analysis of variance). Multiple comparisons analysis revealed significant differences between the $\mathrm{HH}$ grade II and IV + V groups and between the $\mathrm{HH}$ grade III and IV $+\mathrm{V}$ groups $(\mathrm{p}<0.05)$ (Fig. 2A). The MTT was significantly prolonged as neurological severity increased, indicating significant grade dependence ( $p<0.05$, analysis of variance). Multiple comparisons analysis revealed a significant difference between the HH grade II and IV + V groups $(\mathrm{p}<0.05)$ (Fig. 2B). The CBV also showed significant grade dependence ( $p<0.05$, analysis of variance). Multiple comparisons analysis revealed no significant difference between the $\mathrm{HH}$ grade II and IV $+\mathrm{V}$ groups ( $\mathrm{p}$ $=0.085$ ) (Fig. 2C).

The relationships between outcome and the hemodynamic parameters CBF, MTT, and CBV were assessed. Outcome worsened as CBF decreased, indicating significant CBF dependence ( $p<0.05$, analysis of variance). CBF was significantly higher in the GR + MD group (33.1 \pm $10.9 \mathrm{ml} / 100 \mathrm{~g} / \mathrm{min}$ ) than in the SD + VS + D group $(24.7 \pm 8.4 \mathrm{ml} / 100 \mathrm{~g} / \mathrm{min})(\mathrm{p}<0.05)$ (Fig. 3A). Outcome worsened as MTT was prolonged, indicating significant MTT dependence ( $p<0.05$, analysis of variance). The MTT was significantly longer in the $\mathrm{GR}+\mathrm{MD}$ group $(6.2 \pm 0.8 \mathrm{sec})$ than in the SD + VS $+\mathrm{D}$ group $(7.7 \pm 2.2 \mathrm{sec}$ ) (Fig. $3 \mathrm{~B})$. Outcome worsened as CBV decreased, indicating that outcome was not dependent on CBV ( $p=0.267$, analysis of variance). CBV was not significantly higher in the 

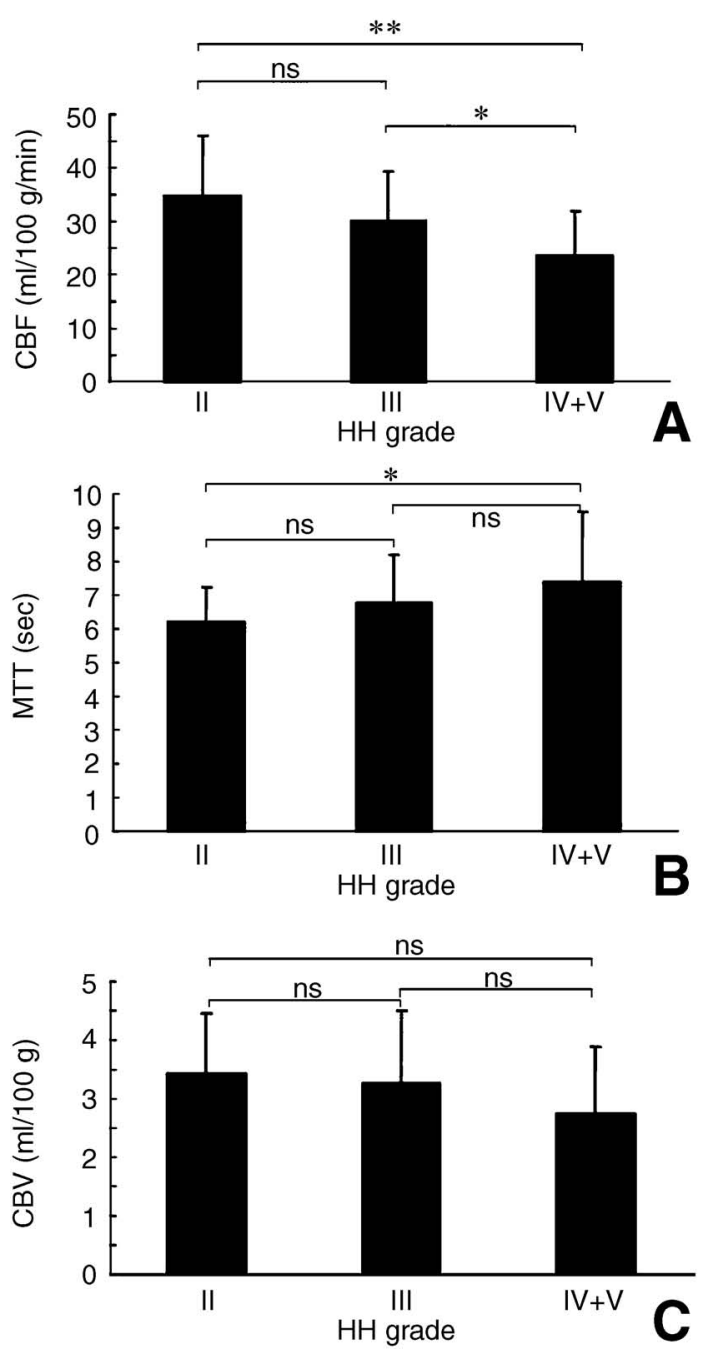

Fig. 2 A: Cerebral blood flow (CBF) was compared in patients with Hunt and Hess (HH) grades II, III, and IV + V. Analysis of variance showed significant differences in CBF. Multiple comparisons analysis of $\mathrm{CBF}$ showed significant differences between $\mathrm{HH}$ grades II and $\mathrm{IV}+\mathrm{V}\left({ }^{* *} \mathbf{p}<\mathbf{0 . 0 1}\right)$, and between $\mathrm{HH}$ grades III and IV + V $\left({ }^{*}\right.$ p $\left.<0.05\right)$. B: Mean transit time (MTT) was compared in patients with HH grades II, III, and IV + V. Analysis of variance showed significant differences in MTT. Multiple comparisons analysis of MTT showed significant differences between $\mathrm{HH}$ grades II and IV + V $\left({ }^{*}\right.$ p $\left.<0.05\right)$. C: Cerebral blood volume (CBV) was compared in patients with HH grades II, III, and IV + V. Analysis of variance showed no significant differences in CBV. Multiple comparisons analysis of CBV showed no significant differences between $\mathrm{HH}$ grades II and IV $+V(p=0.085)$. ns: not significant.

GR + MD group $(3.3 \pm 1.2 \mathrm{ml} / 100 \mathrm{~g})$ than in the SD $+\mathrm{VS}+\mathrm{D}$ group $(3.0 \pm 1.2 \mathrm{ml} / 100 \mathrm{~g})(\mathrm{p}=0.30)($ Fig. $3 \mathrm{C})$.

Discriminant analysis of the above three
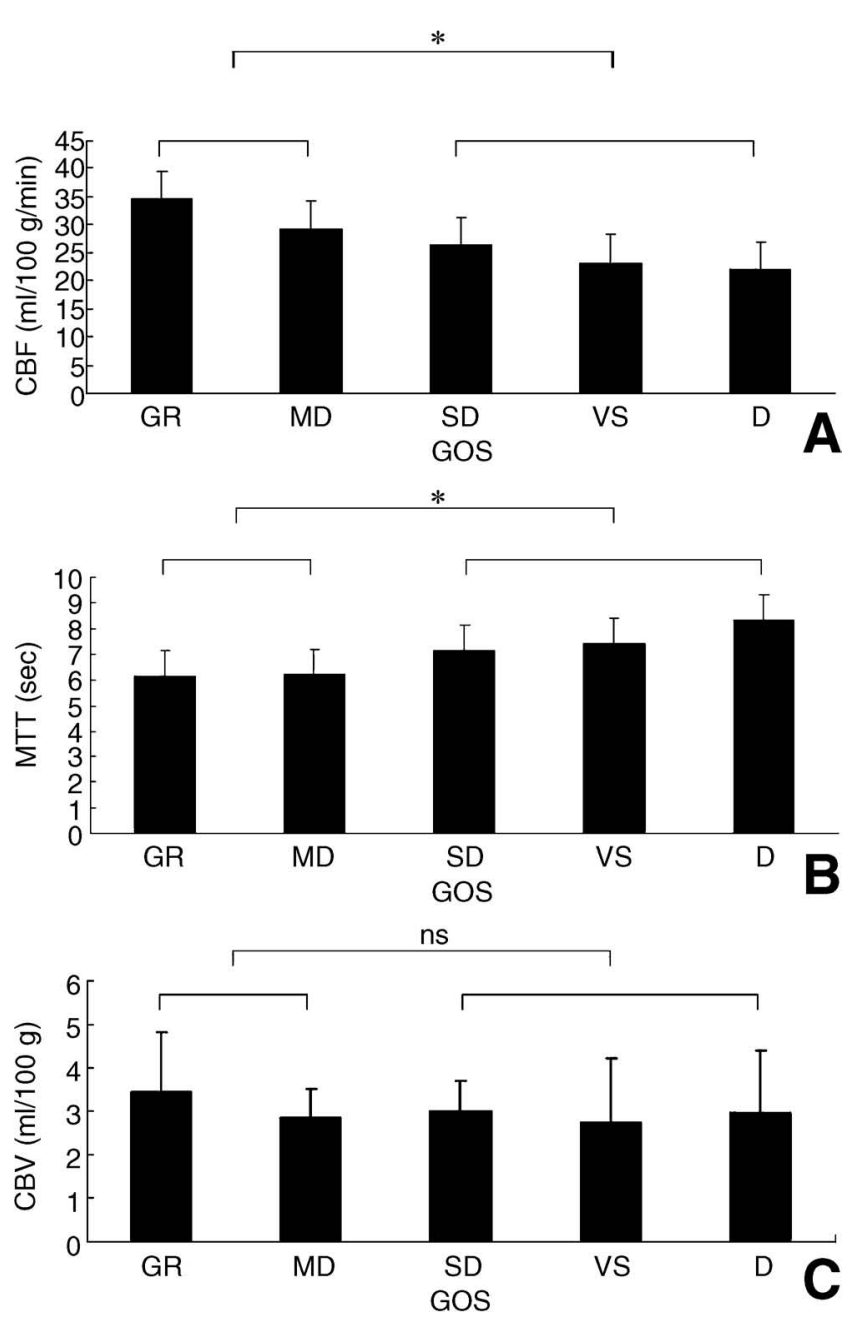

Fig. 3 A: Analysis of variance for cerebral blood flow (CBF) showed significant differences in Glasgow Outcome Scale (GOS). CBF was compared between patients with GOS good recovery (GR) + moderate disability (MD) and severe disability (SD) + vegetative state (VS) + death (D). There was a significant difference between GR + MD and SD + VS + D ( $\left.{ }^{*} p<0.05\right)$. B: Analysis of variance for mean transit time (MTT) showed significant differences in GOS. MTT was compared between patients with GOS GR + MD and SD + VS + D. There was a significant difference between GR + MD and SD + VS + D ( $\left.{ }^{*} \mathbf{p}<\mathbf{0 . 0 5}\right)$. C: Analysis of variance for cerebral blood volume (CBV) showed no significant differences in GOS. CBV was compared between patients with GOS GR + MD and SD + VS + D. There was no significant difference between $G R+M D$ and SD $+V S+D(p=0.300)$. ns: not significant.

parameters showed that MTT and CBF were effective discriminant parameters, whereas CBV was not. Using the discriminant equation $\mathrm{y}=-0.062 \mathrm{CBF}+$ 0.467 MTT -1.342 , we were able to predict outcomes with a probability of $74.5 \%$ (Fig. 4 ). 


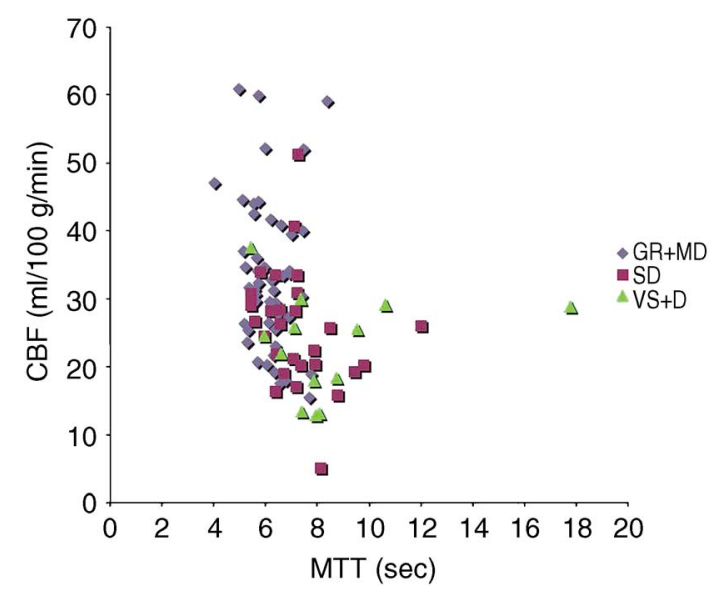

Fig. 4 Scatter plot showing the relationship between cerebral blood flow (CBF) and mean transit time (MTT). The favorable outcome group tended to display increased CBF and decreased MTT. The unfavorable outcome group tended to display decreased CBF and increased MTT. Blue diamonds: good recovery (GR) + moderate disability (MD) cases, purple squares: severe disability (SD) cases, green triangles: vegetative state (VS) + death (D) cases.

\section{Discussion}

Understanding of the cerebral circulation dynamics in the early phase of SAH is important to determine the pathogenesis, develop appropriate treatment plans, evaluate treatment results, and predict outcomes, so many studies have examined this topic. However, many such studies have investigated the cerebral circulation and metabolism during delayed CVS, and few have examined the cerebral circulation and metabolism in the early stage before the development of delayed CVS. ${ }^{5-7,11,20,25,26)}$ The cerebral circulation is impaired from the early stages after SAH onwards and CBF is markedly reduced in severe cases. ${ }^{23)}$ We also confirmed that CBF decreased as the neurological grade increased. Thus, we speculate that such decreases in CBF from the early stages after SAH onset induce severe clinical symptoms and adversely influence the final outcome. Indeed, the present study showed that decreases in CBF and increases in MTT were associated with poor outcome. Thus, we consider that the neurological grade of SAH patients on admission is determined by the severity of their cerebral circulatory disturbance, and that patients suffering from cerebral circulation disturbance before the development of delayed CVS have poor outcome.

Positron emission tomography, single photon emission computed tomography, Xe-CT, and perfusion CT can be used to evaluate cerebral circulation and metabolism in the early phase of SAH, and CT is routinely used for the follow up of SAH. Xe-CT and perfusion CT are convenient because they can be performed successively after standard CT examination. In particular, recent studies have reported the utility of perfusion CT for SAH.8,9,16,28) MTT values obtained from this modality are believed to be much more sensitive than other parameters for evaluating cerebral vasospasm. ${ }^{28)}$ However, CBF values obtained from perfusion CT are not quantitative, although they are correlated with those obtained from Xe-CT. ${ }^{22}$ Based on these reports, we performed Xe-CT and perfusion CT simultaneously to examine whether CBF, CBV, and MTT and maps of these parameters created by image processing are useful for evaluating cerebral circulatory disturbance following SAH and whether they can be used to predict outcomes.

In the present study, significant reduction in CBF and significant MTT prolongation were observed as the neurological grade on admission increased. The finding of MTT prolongation on perfusion CT may suggest cerebral circulatory disturbance. ${ }^{1)}$ This finding may also be useful in the acute phase of cerebral infarction. ${ }^{19,24,27,29)}$ This is in agreement with the changes in CBF and MTT observed during delayed CVS, ${ }^{12,25,28)}$ which are considered to suggest cerebral circulatory disturbance. Also, a threshold MTT value for diagnosing CVS has been reported, but it varies depending on the perfusion CT protocol, software, and hardware used at individual centers. In the present study, we measured the MTT (which was calculated as the distance between two inflection points, ${ }^{18)}$ not from CBF and CBV as in the deconvolution method). The contrast medium injection site, catheter thickness, and infusion rate remained constant. Along with $\mathrm{CBF}$ values, the MTT values obtained under these constant conditions were shown to be quantitative and useful for the evaluation of cerebral circulatory disturbance.

An animal experiment reported that MTT prolongation in the hyperacute phase (day 0) of SAH was associated with high mortality rate, and this was due to ICP elevation in the acute phase. ${ }^{13)}$ The prolongation of the cerebral circulation time (CCT) on digital subtraction arteriography (DSA) has also been reported to reflect increased ICP and to be related to the prognosis. ${ }^{30}$ It appears that, in occlusive cerebrovascular disease, the cerebral perfusion pressure decreases, the CBV increases due to compensatory dilatation of the cerebral blood vessels, and the MTT is prolonged. However, in the acute phase of $\mathrm{SAH}$, the CBV and CBF decrease due to ICP elevation, and the MTT is prolonged. In this study, CBV decreased as the severity of the patient's condition 
increased, although the increase was not significant. The reduced CBF and prolonged MTT detected by Xe-CT and perfusion CT are thought to have been caused by another factor. As described previously, these changes agree with those observed during delayed CVS. In the present study, we measured these parameters in the early phase (Days 1-3) before delayed CVS. This suggests that cerebral circulatory disturbance occurs before delayed CVS. As we did not detect any circulatory disturbance that was consistent with the flow territories of the major vessels, these changes were not thought to be attributable to vasospasm in the major arteries, but rather to represent increased small vessel resistance or the narrowing of small vessels. Therefore, the reduction in CBF and the prolongation of MTT observed before delayed CVS were considered to have been due to cerebral damage caused by microcirculatory disturbance. Indeed, in the present study, a ventricular drainage tube or continuous cisternal drainage tube was inserted into patients being treated for aneurysms; therefore, their ICP was successfully controlled, suggesting that microcirculatory vasoconstriction influences the development of cerebral circulatory disturbance during the early phase of SAH. Not only marked narrowing of the large arteries but also microcirculatory changes, which were indicated by a prolonged CCT, affected cerebral ischemia during cerebral vasospasm. ${ }^{17)}$ Other studies have reported that delayed cerebral ischemia, which develops during delayed CVS, occurred in 11-49\% of patients in whom no vasospasm was found on DSA, ${ }^{2,3,28)}$ suggesting the involvement of microcirculatory vasospasm. The factors involved in the mechanism responsible for the cerebral circulation disturbance that occurs prior to the development of delayed CVS appear to be ICP elevation and microcirculatory vasoconstriction.

Discriminant analysis of $\mathrm{CBF}, \mathrm{MTT}$, and CBV identified MTT and CBF as outcome predictors. Using the discriminant equation $\mathrm{y}=-0.062 \mathrm{CBF}+$ 0.467 MTT -1.342 , we were able to predict outcomes with a probability of $74.5 \%$. CBF was significantly lower in patients with poor outcome than in those with favorable outcome, and MTT was significantly longer in the former than in the latter. Reduction in CBF and MTT prolongation before delayed CVS are considered to represent cerebral circulatory disturbance and to be predictors of poor final outcome.

The results of this study show that cerebral circulatory disturbance following SAH occurs before delayed CVS and can be evaluated using the cerebral circulation parameters CBF and MTT. In addition, we found that it was possible to predict outcomes with reasonable accuracy based on circulatory disturbance at this time point, indicating the usefulness of such evaluations.

\section{References}

1) Araki Y, Furuichi M, Nokura H, Sakai N: [Evaluation of cerebral hemodynamics with perfusion CT]. No To Shinkei 54: 581-588, 2002 (Japanese)

2) Aralasmak A, Akyuz M, Ozkaynak C, Sindel T, Tunoer R: CT angiography and perfusion imaging in patients with subarachnoid hemorrhage: correlation of vasospasm to perfusion abnormality. Neuroradiology 51: 85-93, 2009

3) Binaghi S, Colleoni ML, Maeder P, Uske A, Regli L, Dehdashti AR, Schnyder P, Meuli R: CT angiography and perfusion $\mathrm{CT}$ in cerebral vasospasm after subarachnoid hemorrhage. AJNR Am J Neuroradiol 28: 750-758, 2007

4) Chai WN, Sun XC, Lv FJ, Wan B, Jiang L: Clinical study of changes of cerebral microcirculation in cerebral vasospasm after SAH. Acta Neurochir Suppl 110(Pt 1): 225-228, 2011

5) Frykholm P, Anderson JL, Langstrom B, Persson L, Enblad P: Haemodynamic and metabolic disturbances in the acute stage of subarachnoid haemorrhage. Acta Neurol Scand 109: 25-32, 2004

6) Grote E, Hassler W: The critical first minutes after subarachnoid hemorrhage. Neurosurgery 22: 654661, 1988

7) Gupta R, Crago EA, Gallek M, Horowitz M, Hoffman L, Jovin T, Yonas H: Reduced ipsilateral hemispheric cerebral blood flow at admission is predictive of vasospasm with infarction after aneurysmal subarachnoid hemorrhage. Neurocrit Care 9: 27-30, 2008

8) Harrigan MR, Magnano CR, Guterman LR, Hopkins $\mathrm{LN}$ : Computed tomographic perfusion in the management of aneurysmal subarachnoid hemorrhage: new application of an existent technique. Neurosurgery 56: 304-317, 2005

9) Hoeffner EG, Case I, Jain R, Gujar SK, Shah GV, Deveikis JP, Carlos RC, Thompson BG, Harrigan MR, Mukherji SK: Cerebral perfusion CT: technique and clinical applications. Radiology 231: 632-644, 2004

10) Hunt WE, Hess RM: Surgical risk as related to time of intervention in the repair of intracranial aneurysms. J Neurosurg 28: 14-20, 1968

11) Jakobsen M: Role of initial ischemia in subarachnoid hemorrhage following aneurysm rupture. Acta Neurol Scand Suppl 141: 1-33, 1992

12) Kanazawa R, Kato M, Ishikawa K, Eguchi T, Teramoto A: Convenience of the computed tomography perfusion method for cerebral vasospasm detection after subarachnoid hemorrhage. Surg Neurol 67: 604-611, 2007

13) Laslo AM, Eastwood JD, Pakkiri P, Chen F, Lee TY: CT perfusion-derived mean transit time predicts early mortality and delayed vasospasm after experimen- 
tal subarachnoid hemorrhage. AJNR Am J Neuroradiol 29: 79-85, 2008

14) Lefournier V, Krainik A, Gory B, Derderian F, Bessou P, Fauvage B, Le Bas JF, Payen JF: Perfusion CT to quantify the cerebral vasospasm following subarachnoid hemorrhage. J Neuroradiol 37: 284291, 2010

15) Muizelaar JP, Fatouros PP, Schroder ML: A new method for quantitative regional cerebral blood volume measurements using computed tomography. Stroke 28: 1998-2005, 1997

16) Mustonen $T$, Koivisto $T$, Vanninen, Hanninen $T$, Vapalahti M, Hernesniemi J, Kuikka JT, Vanninen E: Heterogeneity of cerebral perfusion 1 week after haemorrhage is an independent predictor of clinical outcome in patients with aneurysmal subarachnoid haemorrhage. J Neurol Neurosurg Psychiatry 79: 11281133, 2008

17) Nabavi DG, LeBlanc LM, BaxterB, Lee DH, Fox AJ, Lownie SP, Ferguson GG, Craen RA, Gelb AW, Lee TY: Monitoring cerebral perfusion after subarachnoid hemorrhage using CT. Neuroradiology 43: 7-16, 2001

18) Oldendorf WH, Kitano M: Radioisotope measurement of brain blood turnover time as a clinical index of brain circulation. J Nucl Med 8: 570-587, 1967

19) Parsons MW, Pepper EM, Bateman GA, Wang Y, Levi CR: Identification of the penumbra and infarct core on hyperacute noncontrast and perfusion CT. Neurology 68: 730-736, 2007

20) Rosenstein J, Suzuki M, Symon L, Redmond S: Clinical use of a portable bedside cerebral blood flow machine in the management of aneurysmal subarachnoid hemorrhage. Neurosurgery 15: 519525,1984

21) Sase S, Honda M, Kushida T, Seiki Y, Shibata I: Quantitative cerebral blood flow calculation method using white matter lambda in Xenon CT. J Comput Assist Tomogr 26: 471-478, 2002

22) Sase S, Honda M, Machida K, Seiki Y: Comparison of cerebral blood flow between computed tomography and xenon-enhanced computed tomography for normal subjects: territorial analysis. J Comput Assist Tomogr 29: 270-277, 2005

23) Schubert GA, Seiz M, Hegewald AA, Hegewald AA, Manville J, Thome C: Acute hypoperfusion immediately after subarachnoid hemorrhage: a xenon con- trast-enhanced CT study. J Neurotrauma 26: 22252231, 2009

24) Sparacia G, Iaia A, Assadi B, Lagalla R: Perfusion CT in acute stroke: predictive value of perfusion parameters in assessing tissue viability versus infarction. Radiol Med 112: 113-122, 2007

25) Sviri GE, Britz GW, Lewis DH, Newell DW, Zaaroor $\mathrm{M}$, Cohen W: Dynamic perfusion computed tomography in the diagnosis of cerebral vasospasm. Neurosurgery 59: 319-325, 2006

26) Tanaka A, Yoshinaga S, Nakayama Y, Tomonaga M: Cerebral blood flow and the response to acetazolamide during the acute, subacute, and chronic stage of aneurysmal subarachnoid hemorrhage. Neurol Med Chir (Tokyo) 38: 623-630, 1998

27) Wintermark M, Flanders AE, Velthuis B, Meuli R, van Leeuwen $M$, Goldsher D, Pineda C, Serena J, van der Schaaf I, Waaijer A, Nesbit G, Gabriely I, Medina V, Quiles A, Pohlman S, Quist M, Schnyder P, Bogousslavsky J, Dillon WP, Pedraz S: Perfusion-CT assessment of infarct core and penumbra: receiver operating characteristic curve analysis in 130 patients suspected of acute hemispheric stroke. Stroke 37: 979-985, 2006

28) Wintermark M, Ko NU, Smith WS, Liu S, Higashida RT, Dillon WP: Vasospasm after subarachnoid hemorrhage: utility of perfusion CT and CT angiography on diagnosis and management. AJNR Am J Neuroradiol 27: 26-34, 2006

29) Yamada M, Yoshimura S, Kaku Y, Iwama T, Watarai $\mathrm{H}$, Andoh T, Sakai N: Prediction of neurologic deterioration in patients with lacunar infarction in the territory of the lenticulostriate artery using perfusion CT. AJNR Am J Neuroradiol 25: 402-408, 2004

30) Yoshimoto Y, Tanaka Y, Sanada T: Angiographic assessment of cerebral circulation time for outcome prediction in patients with subarachnoid hemorrhage. Surg Neurol 62: 115-120, 2004

Address reprint requests to: Mitsuru Honda, MD, PhD, Department of Critical Care Center, Toho University Medical Center Omori Hospital, 6-11-1 OmoriNishi, Ota-ku, Tokyo 143-8541, Japan.

e-mail:mhonda@toho-u.ac.jp 